\title{
Ensino da Odontologia Hospitalar no Sul do Brasil
}

\author{
Bruno Bevenuto Lucas*; José Lázaro Rodrigues Vieira Júnior*; João Felipe Besegato**; Pablo \\ Guilherme Caldarelli***
}

* Estudante do Curso de Graduação em Odontologia, Universidade Estadual de Londrina

** Estudante do Programa de Pós-Graduação em Odontologia, Universidade Estadual de Londrina

*** Docente do Departamento de Medicina Oral e Odontologia Infantil, Universidade Estadual de Londrina

Recebido em 17/01/2017. Aprovado em 09/04/2017.

\begin{abstract}
RESUMO
O objetivo do presente estudo foi apresentar um panorama da inserção da Odontologia Hospitalar nos Projetos Políticos Pedagógicos (PPP) dos cursos de Odontologia das Instituições de Ensino Superior (IES) da região Sul do Brasil. Trata-se de um estudo quantitativo, descritivo e exploratório. Nos sítios eletrônicos dos cursos buscou-se informações referentes à oferta do componente curricular de Odontologia Hospitalar ou equivalente. Foram analisadas variáveis como natureza das instituições (pública ou privada), momento do curso no qual o componente curricular é ofertado (ano/semestre), natureza do ensino (teórico, prático ou teórico-prático), carga horária total e disponibilidade de ementa, objetivos e conteúdos ministrados. Participaram do estudo 40 cursos de graduação em Odontologia da região Sul do Brasil. Em seis deles não foram encontradas informações do PPP. Com relação à oferta, seis cursos apresentam Odontologia Hospitalar na matriz curricular, dois no Paraná e quatro no Rio Grande do Sul, todas instituições privadas. Quanto ao momento de oferta e a carga horária, em três encontra-se no décimo semestre com $65 \mathrm{~h}$, em uma no nono semestre com $68 \mathrm{~h}$ e em duas estas informações não estavam disponíveis. Todas apresentaram ementas, entretanto, não foram encontradas informações da natureza (teórica/prática) dos componentes curriculares. $\mathrm{O}$ estudo traçou um panorama da inserção da Odontologia Hospitalar nos cursos de Odontologia do Sul do Brasil, apontando a necessidade de discussão acerca da implementação deste conteúdo. Observou-se também que nem todos os cursos disponibilizam informações da matriz curricular online, e quando disponíveis nem todas as características são apresentadas.
\end{abstract}

Descritores: Educação em Odontologia. Ensino. Currículo. Equipe Hospitalar de Odontologia.

\section{INTRODUÇÃO}

A busca pela redução de infecções e doenças sistêmicas causadas pela proliferação de microrganismos presentes na cavidade oral são ações resultantes de trabalho multiprofissional em ambiente hospitalar. Neste cenário, a presença do cirurgião-dentista e a busca por cuidados referentes às condições bucais de pacientes que se encontram neste ambiente é uma nova realidade encontrada em muitas cidades e estados do Brasil, e tem se tornado fundamental para a promoção, prevenção e minimização de 
alterações e manifestações bucais de pacientes hospitalizados $^{1,2}$.

A Odontologia Hospitalar é uma recente área de atuação dos cirurgiões-dentistas, a qual ainda se encontra em discussão em âmbito legal, profissional e científico. De acordo com o Conselho Federal de Odontologia (CFO), não é considerada como uma especialidade odontológica, tratando-se, de acordo com as Resoluções CFO 162/15 e 163/15, de uma área do conhecimento para a qual o cirurgião-dentista pode requerer habilitação ${ }^{3,4}$.

Dentre as áreas de atuação do profissional habilitado em Odontologia Hospitalar destacamse aquelas relacionadas com: a) a atuação em equipes multiprofissionais, interdisciplinares e transdisciplinares na promoção da saúde baseada em evidências científicas; b) a assistência odontológica aos pacientes críticos, em regime de internação, ambulatorial, domiciliar, urgência e emergência; c) a aplicação do conhecimento adquirido na clínica propedêutica, diagnóstico, indicações e uso de evidências científicas; e d) a integração em programas de promoção, manutenção, prevenção, proteção e recuperação da saúde em ambiente hospitalar ${ }^{4}$.

Considerando a discussão sobre a Odontologia Hospitalar em âmbito legal, tornase importante destacar o Projeto de Lei da Câmara nº. 34/2013 que visa à regulamentação da presença do cirurgião dentista nas Unidades de Terapia Intensiva (UTIs) e a assistência odontológica no atendimento e internações domiciliares do Sistema Único de Saúde (SUS) ${ }^{5}$. Esse projeto de lei ainda não foi aprovado e encontra-se no Plenário do Senado Federal, aguardando inclusão na pauta de votação.

Dessa forma, com base no cenário atual da Odontologia Hospitalar no Brasil, o acesso à informação e ao conhecimento, quanto ao atendimento e abordagens específicas a esse público alvo, vem crescendo, se disseminando e adquirindo um espaço e apoio significativos das demais classes profissionais ${ }^{1,6}$. Diante deste contexto, torna-se fundamental que as Instituições de Ensino Superior (IES) com curso de Odontologia adequem suas matrizes curriculares com conteúdos relacionados à prática da Odontologia Hospitalar, proporcionando aos futuros cirurgiões-dentistas o desenvolvimento de habilidades e competências necessárias para o atendimento universal, resolutivo, humanizado e de qualidade.

As Diretrizes Curriculares Nacionais (DCN) em Odontologia que definem os objetivos do curso e os princípios na formação de cirurgiões-dentistas destacam-se como facilitadoras para a inserção destes conteúdos curriculares nos cursos de graduação em Odontologia. As DCN devem ter aplicação nacional e constituir o eixo orientador na elaboração dos currículos implementados por todas as IES, sendo complementadas por uma dimensão diversificada e específica, que reflita a experiência, a vocação de cada escola e as necessidades e imposições da região na qual se situa $^{7,8,9}$. Com o intuito de cumprir os pressupostos das DCN, a apresentação dos PPP das IES torna-se imprescindível na determinação dos objetivos do curso, bem como os fundamentos, condições e procedimentos para a formação de cirurgiões-dentistas ${ }^{3}$.

O objetivo do presente estudo foi apresentar um panorama situacional da inserção da Odontologia Hospitalar nos PPP dos cursos de Odontologia da região Sul do Brasil.

\section{MATERIAL E MÉTODO}

Trata-se de um estudo quantitativo, exploratório e descritivo, no qual o objeto do estudo compreendeu os 40 cursos de Odontologia das IES da Região Sul do Brasil, 
segundo informações coletadas no sítio eletrônico do $\mathrm{CFO}^{10}$. Por se tratar de coleta de dados secundários, não houve necessidade de análise do projeto por Comitê de Ética em Pesquisa.

Foram analisados todos os sítios eletrônicos dos cursos de Odontologia das IES da região Sul do Brasil, buscando informações nos PPP sobre a inserção e oferta do componente curricular de Odontologia Hospitalar ou equivalente nos currículos. Foram analisadas também a natureza das instituições (pública ou privada), o local das instituições, o momento do curso no qual o componente curricular é oferecido (ano/semestre), a natureza do ensino (teórica, prática ou teórico-prática), a carga horária total e a disponibilidade da ementa, objetivos e conteúdos ministrados.

Os dados coletados foram tabulados pelo software Microsoft Excel® e tratados por estatística descritiva.

\section{RESULTADOS}

No momento da coleta de dados foram encontradas 40 IES da Região Sul do Brasil que ofertam o curso de Odontologia, das quais 11 $(27,5 \%)$ são públicas e $29(72,5 \%)$ são privadas (figura 1). Dessas 40 IES, apenas seis (18\%) apresentaram o componente curricular de Odontologia Hospitalar em seu PPP, sendo todas instituições privadas (figura 2). Torna-se importante ressaltar que, durante a coleta de dados do presente estudo, no sítio eletrônico de algumas IES os PPP não estavam disponíveis. Dessa forma, não foram encontradas informações referentes à seis instituições de ensino, sendo uma IES pública e cinco IES privadas.

A distribuição geográfica das IES do Sul do Brasil que apresentam o componente curricular de Odontologia Hospitalar em seus PPP encontra-se esquematizada na figura 3.

Quanto ao momento do curso no qual o componente curricular é ofertado (ano/semestre), natureza (teórica, prática ou teórico-prática) e carga horária total, os resultados obtidos mostraram que dentre as seis IES privadas, duas instituições situadas no Paraná (PR) ofertam o componente curricular, entretanto não relatam as informações específicas.

Em três instituições localizadas no Estado do Rio Grande do Sul (RS), o componente curricular se encontra no décimo semestre, apresentando uma carga horária total de 68 horas, não sendo informada no PPP a natureza do ensino. Uma IES do estado do RS oferece o componente curricular durante o nono semestre do curso, com carga horária de 85 horas, entretanto, não foram encontradas informações relacionadas à natureza do ensino.

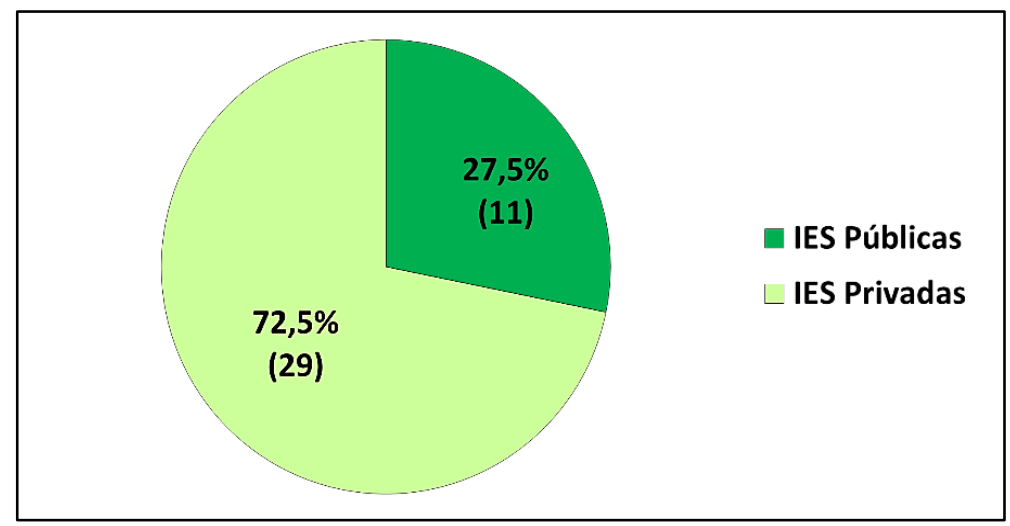

Figura 1 - Natureza das IES da região Sul do Brasil que oferecem curso de Odontologia 


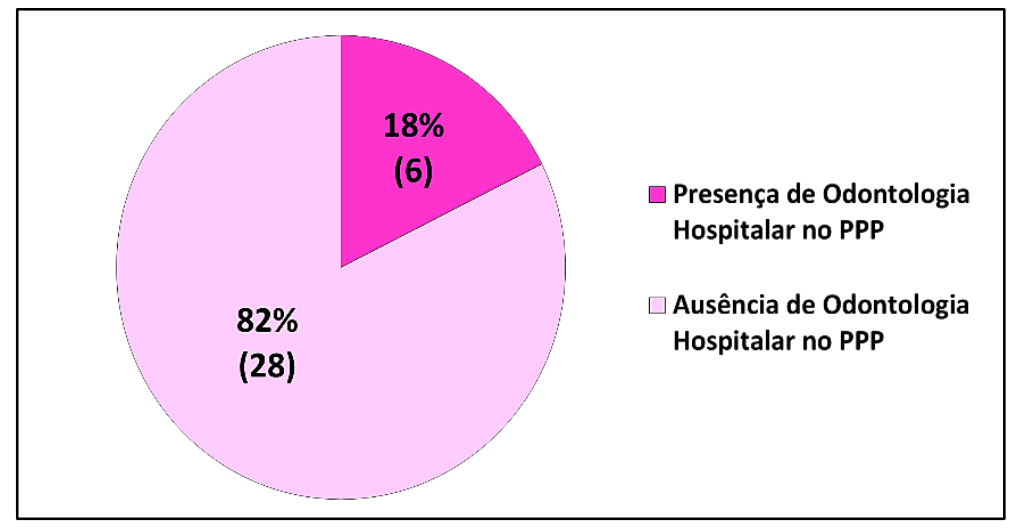

Figura 2 - Presença e ausência do componente curricular de Odontologia Hospitalar nos PPP dos cursos de Odontologia do Sul do Brasil

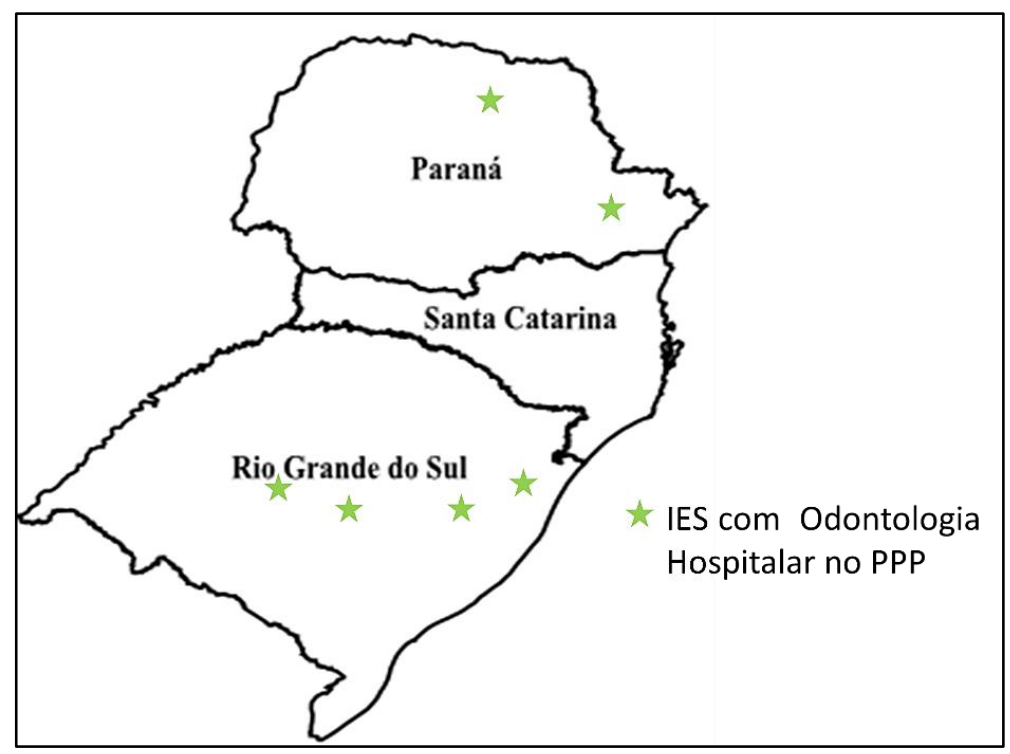

Figura 3 - Distribuição geográfica das IES do Sul do Brasil que apresentam o componente curricular de Odontologia Hospitalar em seus PPP

\section{DISCUSSÃO}

Atualmente, a prática odontológica vem sendo introduzida nos hospitais ${ }^{1,11}$. Neste cenário, os cirurgiões-dentistas têm, cada vez mais, buscado formação específica e adequada para a realização de procedimentos que devem ser realizados neste ambiente ${ }^{11}$. Assim, na medida em que a Odontologia é inserida nos hospitais, estes recursos devem ser utilizados, aprimorados e valorizados, pois diversos tratamentos são inviáveis de realização em consultórios odontológicos, necessitando dos recursos tecnológicos e também da integração multidisciplinar $^{11,12}$. Para isso, torna-se necessário que os profissionais estejam conscientes de que as especialidades necessitam estar interligadas, proporcionando um atendimento integrado que vise o bemestar geral dos pacientes ${ }^{11}$.

A importância e o impacto dos profissionais de Odontologia no que diz respeito à saúde geral dos pacientes em 
tratamento hospitalar e à qualidade dos serviços prestados são conhecidas ${ }^{2,13}$. O cirurgião-dentista deve estar preparado para atuar nos diversos cenários em nível hospitalar, como internações, solicitações e interpretação de exames complementares e controle de infecções que auxilia de forma direta na diminuição de custos e na média de permanência do paciente no hospital ${ }^{2,14}$. Essas práticas, no entanto, sempre foram colocadas às margens das políticas públicas de saúde e ainda enfrentam dificuldades significativas para se efetivarem nas rotinas de grandes centros de saúde.

Uma das maneiras encontradas para adequar as práticas e a atuação dos cirurgiõesdentistas em ambientes hospitalares encontrase relacionada com a oferta de cursos em nível de habilitação e residências odontológicas. Entretanto, em termos de graduação em Odontologia, a presença do componente curricular de Odontologia Hospitalar nos currículos das instituições de ensino superior ainda se encontra bastante discreta ${ }^{1}$. Neste contexto, destaca-se a importância dos estudantes de graduação em Odontologia, como futuros cirurgiões-dentistas, serem incentivados e preparados, durante a sua formação acadêmica, para o manejo adequado do paciente em nível hospitalar ${ }^{1,11,12}$.

Assim, as instituições de ensino superior com curso de Odontologia devem conscientizar os alunos da importância da inserção do componente curricular de Odontologia Hospitalar para o desenvolvimento de habilidades e competências necessárias para o manejo adequado desses pacientes, objetivando o atendimento universal, resolutivo, humanizado e de qualidade $^{1}$; proporcionando a capacitação e o conhecimento teórico-prático necessários aos estudantes.
As DCN dos cursos de graduação em Odontologia foram instituídas pelo Conselho Nacional de Educação (2002), incentivadas pela necessidade de mudança de um modelo pedagógico vigente, hegemônico, baseado em um paradigma biologista, centrado em conteúdos isolados, dissociando os conhecimentos e fragmentando o ser humano ${ }^{9,15}$. As DCN possibilitam uma maior flexibilidade dos currículos, contribuindo para um ensino-aprendizado dinâmico, que contribua para a formação de um profissional humanista, crítico, reflexivo e capaz de atuar nos diversos cenários e níveis de atenção ${ }^{7-9,16}$. Dessa forma, mudanças curriculares voltadas para a implementação do componente curricular de Odontologia Hospitalar e afins, estariam em consonância com os pressupostos das Diretrizes Curriculares em Odontologia.

A discussão a respeito da inserção desse componente curricular nas matrizes curriculares dos cursos de Odontologia deve ocorrer com o intuito de aumentar a participação e a atuação dos cirurgiõesdentistas no ambiente hospitalar, mostrando aos estudantes e à população a importância de melhorar o atendimento dos pacientes hospitalizados $^{1}$ e a necessidade de uma formação profissional alicerçada nos princípios de integralidade e multiprofissionalismo. Neste cenário, a Associação Brasileira de Ensino Odontológico (ABENO) juntamente com Sociedade Brasileira de Estomatologia e Patologia Oral (SOBEP) têm discutido como a área de Odontologia Hospitalar e a especialidade de Estomatologia podem estar em consonância com o processo de formação do profissional para atuação no ambiente hospitalar. Essa discussão curricular tem destacado que as atividades desenvolvidas por esses profissionais são complexas e exigem conhecimentos específicos muitas 
vezes não inseridos nos currículos dos cursos de graduação em Odontologia, tornando necessário se estabelecer novas habilidades e competências, como ratificado pelo presente estudo $^{17}$.

Os resultados obtidos evidenciam a ausência do componente curricular de Odontologia Hospitalar nos PPP na grande maioria (82\%) das IES da região Sul do Brasil. Considerando o atual cenário e as limitações do estudo, tais dados devem ser melhor investigados, considerando formas alternativas de coleta de dados, como a utilização de questionários ou entrevistas com dirigentes das IES, aprimorando a compreensão desse panorama.

\section{CONCLUSÃO}

O presente estudo possibilitou traçar um panorama da inserção da Odontologia Hospitalar nos PPP das IES do Sul do Brasil, mostrando que a implementação do componente curricular nos cursos de Odontologia se encontra bastante discreta e concentrada apenas em IES privadas. Foi possível observar também que nem todos os cursos disponibilizam informações sobre sua organização curricular online, e quando estão disponíveis, nem todas as características são apresentadas.

Dessa forma, os resultados encontrados reafirmam a necessidade da elaboração de novos trabalhos, utilizando outros métodos de coleta de informações, com a finalidade de aprimorar a compreensão da temática e destacando a importância do componente curricular de Odontologia Hospitalar na formação de cirurgiões-dentistas.

\section{ABSTRACT \\ Hospital Dentistry Teaching In Southern Brazil}

The aim of this study was to present an overview of the inclusion of hospital dentistry in the Pedagogic Political Project (PPP) of Dentistry courses of Higher Education Institutions (HEI) from Southern Brazil. This is a quantitative, descriptive and exploratory study. The courses' websites were analyzed seeking information related to the offer of hospital dentistry curriculum component or equivalent. It was also examined the nature (public or private), and the State of the institutions, the period in which the course is offered (year/semester), nature of education (theoretical, practical or theoretical-practical), total workload, availability of menu, objectives, and contents taught. 40 undergraduate courses in Dentistry of the southern of Brazil participated in the study. In six of them were not found PPP information. Regarding the offer, six courses have Hospital Odontology in the curriculum, two in Paraná State and four in the Rio Grande do Sul State, all private institutions. About the period in which the course is offered and total workload, in three courses the discipline is in the tenth semester with 65 hours, in one course is in the ninth semester with 68 hours, and in two courses this information was not available. No information was found about nature (theoretical/practical) of the curriculum component. The study outlined the insertion of Hospital Dentistry in the Southern Brazil Dentistry courses, indicating the need for discussion about the implementation of the curriculum component. Also, it was observed that not all dental courses provide information about their curriculum online, and when available, not all features are presented.

Descriptors: Education, Dental. Teaching. Curriculum. Dental Staff, Hospital.

\section{REFERÊNCIAS}

1. Aranega AM, Bassi APF, Ponzoni D, Wayama MT, Esteves JC, Júnior IRG. Qual a importância da Odontologia Hospitalar? Rev Bras Odontol. 2012; 69(1):90-3.

2. Pinheiro TS, Almeida TF. A saúde bucal em pacientes de UTI. Rev Bahiana Odonto. 
2014;5(2):94-103.

3. Conselho Federal de Odontologia (Brasil). Resolução $n^{\circ}$. 162, de 03 de novembro de 2015. Reconhece o exercício da Odontologia Hospitalar pelo cirurgiãodentista. Diário Oficial da União 16 nov 2015;Seção 1. [Acesso em 08 abr. 2017]. Disponível em: http://cfo.org.br/wpcontent/uploads/2015/12/ResolucaoCFO162-15.pdf.

4. Conselho Federal de Odontologia (Brasil). Resolução $n^{\circ}$. 163, de 09 de novembro de 2015. Conceitua a Odontologia Hospitalar e define a atuação do cirurgião-dentista habilitado a exercê-la. Diário Oficial da União 25 nov 2015. [Acesso em 08 abr. 2017]. Disponível em https://www. legisweb.com.br/legislacao/?id=310456.

5. Brasil. Senado Federal. Projeto de lei $\mathrm{n}^{\circ} 34$ de 2013. Torna obrigatória a prestação de assistência odontológica a pacientes em regime de internação hospitalar, aos portadores de doenças crônicas e, ainda, aos atendidos em regime domiciliar na modalidade home care [Internet]. [Acesso em 08 abr. 2017]. Disponível em: https://www25.senado.leg.br/web/atividad e/materias/-/materia/112975.

6. Paulino GS. Importância do cirurgiãodentista na equipe multidisciplinar em ambiente hospitalar [Monografia]. Paraíba: Universidade Estadual da Paraíba (UFPB). 2016. [Acesso em 17 jan. 2017]. Disponível em http://dspace.bc.uepb. edu.br/jspui/handle/123456789/9930.

7. Brasil. CNE. Parecer CES/CNE 1300/2001 [internet]. Diário Oficial da União, Seção 1, p. 25; Brasília, 7 de dezembro de 2001. [Acesso em 17 jan. 2017]. Disponível em: http://portal.mec.gov.br/cne/arquivos/pdf/ CES1300.pdf.

8. Brasil. CNE. Resolução CNE/CES 3/2002 [internet]. Diário Oficial da União, Seção 1, p.10; Brasília, 4 de março de 2002. [Acesso em 17 jan. 2017]. Disponível em: http://portal.mec.gov.br/cne/arquivos/pdf/ CES032002.pdf.

9. Ogawa D, Higasi MS, Caldarelli PG. Odontogeriatria nos Projetos Políticos Pedagógicos dos cursos de Odontologia do sul do Brasil. Rev ABENO. 2015;15(4):919.

10. Conselho Federal de Odontologia - CFO [internet]. Consulta Faculdades de Odontologia por Estado. [Acesso em 17 jan. 2017]. Disponível em: http://cfo. org.br/servicos-e-consultas/\%20faculdades/.

11. Godoi APT, Francesco AR, Duarte A, Kemp APT, Silva-Lovato $\mathrm{CH}$. Odontologia hospitalar no Brasil. Uma visão geral. Rev Odontol UNESP. 2009;38(2):105-9.

12. Doro GM, Fialho LM, Losekann M, Pfeiff DN. Projeto "Odontologia Hospitalar". Rev ABENO. 2006;6(1):49-53.

13. Dantas BO, De Araújo IA, De Araújo HBN, De Araújo EC, Bezerra ACB, Miranda AF. Saúde bucal e cuidados na Unidade de Terapia Intensiva. R Odontol Planal Cent. 2015;5(1):28-32.

14. Queluz DP, Palumbro A. Integração do odontólogo no serviço de saúde em uma equipe multidisciplinar. JAO. 2000; 3(19):40-6.

15. Finkler M, Caetano JC, Ramos FRS. Integração "ensino-serviço" no processo de mudança na formação profissional em Odontologia. Interface Comunic Saúde Educ. 2010;15(39):1053-70.

16. Fonseca EP. The National Curriculum Guidelines and training of Brazilian dentists. J Manag Prim Health Care. 2012;3(2):158-78.

17. $50^{\circ}$ Reunião da Associação Brasileira de 
Ensino Odontológico. 2015 ago 12-14; Salvador, Bahia, Brasil [internet]. [Acesso em 08 abr. 2017]. Disponível em: http://reuniaoanual.abeno.org.br/50reunia o/files/ementas/reuniao-sobep.pdf.
Correspondência para:

Pablo Guilherme Caldarelli

e-mail: pablocaldarelli@hotmail.com Rua Juiz de Fora, $n^{\circ}$ 230, Jardim Champagnat 86062-680, Londrina, PR 